\title{
El tratamiento del suicidio en la prensa española: ¿efecto werther o efecto papageno?
}

The treatment of suicide in the spanish press: ¿werther effect or papageno effect?

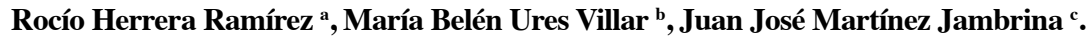 \\ ${ }^{a}$ Psiquiatra. ${ }^{b}$ Psicólogo clínico, ${ }^{c}$ Psiquiatra, Director AGC Salud Mental. ${ }^{a, b y c}$ Servicio de Salud Mental. \\ Hospital San Agustín. Avilés, Asturias, España.
}

Correspondencia: Rocío Herrera Ramírez (sinestesy@yahoo.com.mx)

Recibido: 27/02/2014; aceptado con modificaciones: 23/07/2014

\begin{abstract}
RESUMEN: Introducción: El suicidio causa un millón de muertes cada año en todo el mundo. En España fallecieron 3180 personas en 2011 por este motivo.

Los medios de comunicación ejercen gran influencia sobre las actitudes, creencias y comportamientos de la comunidad. Entre los medios de comunicación, la prensa es calificada como el medio de mayor influencia. Una considerable cantidad de literatura sugiere que los medios de comunicación podrían inducir a actos de imitación del suicidio, este fenómeno se conoce como efecto Werther. Por el contrario, el contenido de las noticias también puede constituir un efecto preventivo, denominado efecto Papageno. La O.M.S. ha elaborado unas recomendaciones dirigidas a los medios de comunicación como parte del programa de prevención del suicidio (SUPRE).

Objetivo: En el presente estudio se analiza si la prensa tiene en cuenta las recomendaciones de la OMS para la publicación de las noticias sobre el suicidio.

Metodología: Se recogen las noticias relacionadas con el suicidio aparecidas en la edición digital de cinco diarios españoles, (El País, El Mundo del Siglo Veintiuno, ABC, El Comercio de Asturias y La Nueva España), desde el 01/09/2011 hasta el 30/10/2012.

Resultados: En la mayor parte de las noticias se dan detalles sobre el método; no se tienen en cuenta los antecedentes en salud mental; no se informa sobre ayudas y recursos comunitarios; se informa acerca del suicidio como algo inexplicable o simplista.

Conclusiones: La mayoría de las noticias recogidas no tienen en cuenta las directrices de la OMS.

PALABRAS CLAVE: Efecto Werther, suicidio, medios de comunicación, prevención.
\end{abstract}

ABSTRACT: Background: Suicide is the cause of death of one million per year. In Spain , 3180 people has been died for this reason in 2011. People are unaware of the risk factors associated with suicide. The media have a great influence on the attitudes, beliefs and behaviors in society. Among the media, the press is considered the most influential. A considerable amount of literature suggest that the media could lead to acts to imitation suicide. This kind of phenomenon is known as "Werther effect" or "Copycat". On the other hand, the content of the news could have a preventive effect called "Papageno effect ". The WHO (World Health Organization) has developed a series of recommendations to the media as part of the program "suicide prevention" (SUPRE).

Aim: This current study analyse whether the press has considered the recommendations of the WHO to publish news about suicide.

Methodology: News about suicide of five spanish newspapers (El País, El Mundo del Siglo Veintiuno, ABC, El Comercio de Asturias y La Nueva España) were collected during a year. From 01/09/2011 to 30/10/2012.

Results: Most news give details about of used method; background in mental health are not taken into account; there are no reports on aid and community resources; sometimes they consider suicide something unexplainable.

Conclusions: Most part of the newspaper don't have into account the WHO recommendations for publish news abut suicide.

KEY WORDS: Werther effect, suicide, media, prevention. 


\section{Introducción}

El suicidio es, actualmente, uno de los mayores problemas de salud pública. Es la causa de alrededor de un millón de muertes cada año a nivel mundial, y en España se ha constituido en la primera causa externa de defunción, se entienden como causas externas de defunción las que son debidas a accidentes, caídas, agresiones o suicidios).

Según el Instituto Nacional de Estadística en 2011 han fallecido 3180 personas en el país por éste motivo. Superando con creces el número de muertes por homicidio (344) dentro del que se incluyen 66 mujeres asesinadas, víctimas de violencia de género (1).

Teniendo en cuenta lo anterior, se puede deducir que el suicidio es un problema que requiere nuestra atención. Desgraciadamente, su prevención y control no son tarea fácil. Investigaciones recientes indican que la prevención del suicidio, si bien es posible, comprende una serie de actividades que van desde la provisión de las mejores condiciones posibles para la educación de jóvenes y niños y el tratamiento eficaz de trastornos mentales, hasta el control medioambiental de los factores de riesgo. La difusión apropiada de información y una campaña de sensibilización del problema son elementos esenciales para el éxito de los programas de prevención.

El suicidio parece ser un fenómeno susceptible de ser imitado. Existe una considerable cantidad de literatura que sugiere que los medios de comunicación al tener un papel significativo en la sociedad y ejercer fuerte influencia sobre las actitudes, creencias y comportamientos de la comunidad, así como jugar un importante rol en la política, la economía y la práctica social, podrían tener una actuación determinante tanto en la prevención como en la conducta imitativa del mismo (2).

Una de las primeras asociaciones conocidas entre el suicidio y los medios de comunicación surgió de la novela de Goethe: "Leiden des jungen Werther" (Las penas del joven Werther), publicada en 1774. En ésta obra, el joven protagonista se suicida disparándose tras un amor infortunado (3-4). Poco después de la publicación del libro se informó acerca de varias personas que usaron el mismo método para suicidarse. Se estableció una relación causal entre estos hechos, dado que muchas veces el libro era encontrado en la escena, la persona iba vestida con el mismo atuendo del protagonista del libro, o hacía referencia al texto en la nota de despedida. Esto causó la prohibición del libro en varios países de Europa.

De aquí proviene el término "efecto Werther", acuñado por el sociólogo David Phillips en 1974, quien tras realizar un estudio entre 1947 y 1968 concluyó que el número de suicidios en Estados Unidos se incrementaba al mes siguiente de que el diario New York Times publicara en portada alguna noticia sobre suicidio (5).

El "efecto Werther" también se conoce como "efecto Copycat" y, según algunos estudios realizados, es más probable que se produzca cuando el modelo es una persona célebre (6). 
Después de éste primer estudio diversos autores e investigadores han llevado a cabo otros en los que se relaciona de manera causal la exposición de la comunidad a cierto tipo de información sobre el suicidio por parte de los medios de comunicación (principalmente prensa) y el posterior aumento de las tasas de tentativa y suicidio consumado.

Los suicidios que con mayor probabilidad atraen la atención de los medios son aquéllos que se apartan de los patrones usuales. Los casos presentados son casi invariablemente atípicos y fuera de lo común y representarlos como típicos perpetúa aún más la información errónea sobre el suicidio.

Clínicos e investigadores reconocen que en la mayoría de los casos existe un cubrimiento sensacionalista del suicidio lo que podría aumentar el comportamiento suicida en poblaciones vulnerables (7).

A la inversa, el cubrimiento responsable, siguiendo unas pautas establecidas, puede tener un efecto preventivo sobre la conducta suicida. A éste efecto se le conoce como "Efecto Papageno", acuñado en honor al personaje homónimo de la "Flauta mágica" de Mozart, que fue disuadido de suicidarse después de que tres niños le mostraran las otras alternativas que le ofrecía la vida. Según el autor Niederkrotenthaler (8), la exposición a información sobre personas afectadas que han afrontado una situación de crisis de manera positiva y sin comportamientos suicidas, se relaciona con un descenso en las tasas de suicidio, ejerciendo, en estos casos, la información, un efecto protector. Concretamente, concluye en su investigación: "El impacto de la información sobre el suicidio no puede limitarse a los efectos nocivos, sino que la cobertura de afrontamiento positivo en circunstancias adversas, como se explica en los artículos de prensa sobre la ideación suicida, pueden tener efectos protectores".

Debido a la controversia existente en la literatura entre la evidencia hallada a favor del efecto Werther, y la encontrada con respecto al efecto Papageno, diversos países han decidido elaborar guías que promueven el reporte responsable de las noticias sobre el suicidio. La mayoría están basadas en las recomendaciones creadas en el año 2000 por parte de la OMS dentro del programa SUPRE (SUICIDE PREVENTION); una iniciativa mundial para la prevención del suicidio lanzada por dicha organización en 1999 y que incluye seis medidas básicas: Tratamiento de las enfermedades mentales, disminución de tóxicos en el gas de vehículos y en el doméstico, control de la posesión de armas de fuego, control sobre la disponibilidad de sustancias tóxicas y disminución de las noticias sensacionalistas sobre suicidios en los medios de difusión (7, 9-10).

A continuación se resumen las recomendaciones del instrumento elaborado para los profesionales de los medios de comunicación, teniendo en cuenta tanto lo que se debe hacer como lo que no: 
- Trabajar estrechamente con autoridades de la salud en la presentación de los hechos.

- Referirse al suicidio como un hecho logrado, no como algo exitoso.

- Presentar solo datos relevantes en las páginas interiores

- Resaltar las alternativas al suicidio

- Proporcionar información sobre líneas de ayuda y recursos comunitarios

- Publicitar indicadores de riesgo y señales de advertencia

- No publicar fotografías o notas suicidas

- No informar de detalles específicos sobre el método usado

- No dar razones simplistas

- No glorificar ni tratar de forma sensacionalista el suicidio

- No usar estereotipos religiosos o culturales

- $\quad$ No aportar culpas (10)

Este trabajo tiene como objetivos principales: describir la manera como la prensa española aborda el tema sobre el suicidio a través del análisis del contenido de las noticias publicadas, y describir si el contenido de la información se ajusta a las recomendaciones dadas por la OMS

\section{Material y método}

\section{Selección de la Muestra}

Para el estudio fueron seleccionados cinco diarios. Los tres de mayor difusión en España según la Oficina de Justificación de la Difusión; El País, El Mundo del Siglo Veintiuno y ABC y los dos de mayor difusión en Asturias; El Comercio de Asturias y La Nueva España.

Se eligieron las ediciones digitales y se analizaron de forma diaria buscando en la hemeroteca virtual de cada una de ellas mediante los siguientes términos: "suicidio", "suicidarse", "intento de suicidio", "tentativa de suicidio"; "matarse", "autolesión" y "autolesionarse".

El período de análisis fue de un año, del 1 de septiembre de 2011 al 30 de septiembre de 2012.

Unidades de Análisis

Como unidad de análisis se seleccionó toda noticia cuyo título o subtítulo contuviera los términos descritos.

Se excluyeron tres noticias que utilizaban los términos de búsqueda como recurso literario (P.ej. "Aceptar el rescate es un suicidio") o de estilo. 
ORIGINALES Y REVISIONES

Registro de datos y análisis estadístico

Para el registro de cada unidad de análisis (noticia seleccionada) se elaboraron cinco variables identificativas y once descriptivas, con éstas se conformó la base de datos.

Se realiza un análisis descriptivo de la frecuencia y porcentaje de aparición de cada una de las variables.

Variables identificativas

Periódico

Cinco grupos: El País, El Mundo del Siglo Veintiuno; ABC; La Nueva España y El Comercio de Asturias

Número de noticias por mes

Se establecieron 13 grupos en función de cada uno de los meses incluidos en el análisis.

Esta variable se introdujo para valorar la constancia o inconstancia de aparición de noticias relacionadas con suicidio a lo largo del tiempo.

Tipo de noticia

Cinco grupos:

Científico-divulgativa: noticias que informaban sobre estudios acerca de suicidios publicados en revistas científicas.

Informe de caso: noticias que detallaban suicidios de personas concretas, fuesen o no celebridades.

Opinión: Artículos sobre opiniones personales acerca del suicidio. En todos los casos fueron elaborados por periodistas que colaboran regularmente con el diario.

Suicidio otros: Artículos que hacían referencia al suicidio sin ser de opinión ni utilizar el término como recurso literario (Promoción de libros y eventos acerca del suicidio y la conducta suicida)

Crónica: Solo una, en el diario ABC, sobre Hitler y los días previos a su muerte Celebridad

Dos grupos: sí, cuando se trataba de un personaje público o famoso (políticos, artistas, deportistas)

No, cuando era una persona anónima

Foto o vídeo

Dos grupos: $\mathrm{Si}$, cuando aparecían en la noticia fotografías o vídeos de la víctima, del método empleado o de la escena del suicidio.

No, en ausencia de material gráfico

Variables de contenido

Método

Se establecieron cuatro grupos según si se hacía alusión al método de suicidio o tentativa de suicidio utilizado sin entrar en detalles. Si se daban detalles acerca de 
cómo fue utilizado el método o como lo consiguió la persona. Si no se nombraba el método y No Procede en los casos en que no era el reporte de un caso.

\section{Nota}

Se establecieron tres grupos según si en la noticia se hacía pública la nota de despedida, o no, de la persona que se había suicidado. No procede, cuando no era un informe de caso.

\section{Factores explicativos}

Tres grupos: Causas múltiples, cuando en la noticia se tenían en cuenta varios factores desencadenantes de la conducta suicida.

Causas simples, cuando en la noticia se explicaba el suicidio como el resultado de un solo hecho. Este grupo se subdividió en dos: Crisis económica y otras. La razón principal es que a medida que se hacía el análisis se encontraron varias noticias donde la única explicación era la crisis económica actual. Otras, se referían a una única causa explicativa como abuso sexual, acoso escolar, ciber-acoso o cualquier otro tipo de problema personal.

No explica, no se hace referencia a alguna razón causante

Héroe

Dos grupos, según si en la noticia se glorificaban a las víctimas como mártires y objetos de adulación. O no.

Ayudas y recursos

Dos grupos, según si en la noticia se proporcionaba información acerca de la existencia de recursos de ayuda como líneas telefónicas, grupos de apoyo y servicios de salud mental, o no se facilitaba información alguna en ese sentido.

Se tuvieron en cuenta las científico-divulgativas y las de opinión, dado que se consideró que juegan un papel importante en la información adecuada que puede dar la prensa acerca del suicidio.

\section{Antecedentes en salud mental}

Dos grupos, según si en la noticia se hacía referencia a antecedentes de patología mental de la persona con conducta suicida. $\mathrm{O}$ no.

Red de apoyo

Tres grupos: $\mathrm{Si}$, cuando se hacía alusión a que la persona con conducta suicida tenía apoyos como familia, amigos o algún otro grupo que generara pertenencia. No, cuando no se hacía referencia. No aplica, si la noticia no era un informe de caso.

Sufrimiento familia

Tres grupos: Sí, cuando se hacía referencia al impacto sobre las familias y otros sobrevivientes en términos de sufrimiento psicológico. O cuando se ofrecían recursos de apoyo (líneas telefónicas, grupos) para los mismos.

No, cuando no se hacía referencia.

No procede, cuando la noticia no era un reporte de caso.

Conducta suicida 
Dos grupos. Suicidio consumado, cuando la noticia se refería a conducta suicida cuyo resultado era el suicidio consumado

Tentativa de suicidio, cuando el resultado de la conducta suicida no era la muerte

Indicadores de riesgo

Dos grupos: $\mathrm{Si}$, cuando en la noticia se aludía a señales de advertencia de riesgo suicida o cuando se hablaba de una serie de factores confluentes en un momento determinado que podían considerarse de riesgo.

No, cuando no se hacía referencia.

Sensacionalismo

Dos grupos: $\mathrm{Si}$, cuando en la noticia ahondaba mucho en detalles escabrosos que intentaban producir emociones intensas en el lector.

No, cuando la noticia era más objetiva

\section{Resultados}

En función de los criterios de inclusión, se recogieron 148 noticias, de las cuales el 30,4 \% fueron recogidas en "El Mundo", el 27\% en "ABC", el 21\% en "El País", el 14,8\% en "La Nueva España" y el 6,8 \% en El Comercio.

Los meses en los que aparecen más noticias publicadas son agosto $(14,2 \%)$ y mayo (11,5\%) y en el que menos septiembre de $2011(3,4 \%)$

La mayor cantidad de noticias recogidas son casos concretos, $75,7 \%$. Sólo hay $9,5 \%$ de noticias científico divulgativas.

El 19,6\% de las noticias se refieren a celebridades.

El 5,4\% de las noticias iban acompañadas de fotos o vídeos

Sumadas las noticias en las que se informa del método con y sin detalles, se obtiene un $65,7 \%$ en que se habla de él.

En el $82.4 \%$ de las noticias no se publica nota

El 21\% de las noticias ofrece factores explicativos múltiples . El 39\% refieren causas simplistas, de éstas el 12,7 se refieren a la crisis económica suponiendo el 48,7 $\%$ del total de noticias en las que se incluyen causas simplistas.

En el 7,4\% de las noticias se ensalza al protagonista como héroe.

En el $94.6 \%$ de los sucesos, no se informa de ayudas y recursos. Mental.

En el 92,6\% de las informaciones no se hace referencia a antecedentes en Salud

El 79,1\% de las noticias no informan si existe red de apoyo.

El 81,8\% de los sucesos no dan cuenta del sufrimiento familiar.

El 79\% de las noticias recogidas se refieren a suicidios consumados.

En el 92,6\% de los casos no se informa sobre indicadores de riesgo.

El $40 \%$ de las noticias se incluyen en la categoría sensacionalismo. 


\section{Discusión}

En los resultados que hemos obtenido, lo primero que llama la atención es el escaso número de noticias publicadas acerca del suicidio, únicamente 148, sobre todo si se tiene en cuenta que según el Instituto Nacional de Estadística asciende a 3180 la cantidad de fallecimientos atribuidos a esta causa. Posiblemente factores como el estigma asociado al suicidio y el miedo al efecto contagio contribuyan a explicar su reducida presencia en los medios de comunicación.

El mayor número de noticias hallado en los medios de ámbito estatal comparado con los de ámbito regional podría deberse a que estos últimos prestan mayor atención a la información de Asturias. Se observa una diferencia entre los periódicos de mayor difusión en España con respecto a la cantidad de noticias: mientras que en "El Mundo" se contabilizan 45 noticias relacionadas con el suicidio, en "El País" únicamente se publicaron 31. Las limitaciones del estudio no permiten establecer si se trata de una diferencia significativa aunque sí nos llama la atención. Cabría preguntarse si la tendencia ideológica del periódico puede influir en la decisión de dar cobertura a informaciones sobre el tema del suicidio.

El mes de Agosto de 2012 es en el que se publican más noticias. Al revisar el material recogido se observa que en esta fecha coinciden las muertes de dos personajes famosos, un director de cine y el batería de una banda musical. Al mismo tiempo, comenzó a cobrar fuerza la asociación entre crisis financiera y conducta suicida. Se realizó un gran despliegue informativo acerca de las muertes por suicidio atribuidas a la crisis económica por la que atraviesan varios países europeos, aumentando tanto las informaciones de caso como las noticias que aluden al suicidio sin referirse a casos concretos. En primer lugar, se informó de dos suicidios llevados a cabo en sitios públicos en dos de los países más afectados, Grecia e Italia. Seguidamente, se produjo una avalancha de noticias de suicidios en estos mismos países. En muchas de las noticias se intentó equiparar su situación con la española.

Con respecto a las variables relacionadas con el contenido, esto es, las referidas a las recomendaciones propuestas por la OMS, hemos hallado que la prensa española no las tiene en cuenta en la mayoría de las informaciones publicadas.

Se presenta información acerca del método usado en el $65 \%$ de las noticias y en un porcentaje importante $(27,5 \%)$ se describe detalladamente cómo se adquirió el mismo y cómo fue utilizado. En las investigaciones revisadas sobre el efecto Werther se presenta evidencia de que explicitar estos elementos contribuye a aumentar el número de suicidios en los que se emplean esos métodos en concreto.

Únicamente se describen causas explicativas múltiples en el $21 \%$ de los casos. De hecho, en la mayoría no aparece explicación del suceso. En ocasiones se llega a simplificar tanto la información, que ni siquiera se menciona algo como: "sin causa aparente". En algunas de las noticias la mayor parte del contenido se encuentra en el 
ORIGINALES Y REVISIONES

mismo titular: "se suicida después de matar a su pareja". Cabría esperar que el cuerpo de la noticia ampliase la información, matizando las posibles causas, exponiendo los factores de riesgo y hablando de otras circunstancias que podrían rodear el hecho. Sin embargo no es esto lo que suele encontrarse.

De entre las causas simplistas que hemos hallado, hay una que nos ha llamado la atención especialmente. A partir de agosto aumentó el número de noticias en las que se relacionaba la crisis con el suicidio. La mayor parte de estas noticias se caracterizaban por titulares en los que se atribuía el suicidio a la crisis económica en una relación directa causa-efecto, obviando otros posibles factores explicativos y culpabilizando a bancos y/o a gobiernos de esas muertes. Queremos destacar que en todas las noticias en las que se publica la nota de despedida aparece la crisis como causa del suicidio. A menudo la parte de la nota que se menciona es una sola frase descontextualizada en la que se alude a la situación financiera del sujeto. Todas las noticias en las que se ensalza como héroe a la persona que se suicida $(7,4 \%)$ son las que relacionan el suicidio con la crisis. En algunas de ellas se llega a justificar el comportamiento de la persona, aduciendo que encontrándose en tal situación límite: "Antes que buscar comida en la basura prefiero morir", era la única opción que le quedaba a la persona.

Las variables que hacen referencia al sufrimiento de la familia, la disponibilidad de red de apoyo, la información acerca de posibles ayudas y recursos comunitarios, la presencia de indicadores de riesgo y los antecedentes en salud mental, son las recomendaciones que se incumplen en mayor medida a la hora de publicar noticias sobre suicidio. Llama especialmente la atención la escasa mención que se hace a los antecedentes en salud mental, dado que según la bibliografía revisada se estima que alrededor de un $90 \%$ de las personas que presentan conductas suicidas tienen antecedentes psiquiátricos $(1,7)$. En nuestro estudio se ve reflejada esta variable únicamente en un $5,4 \%$ de las publicaciones.

Al igual que en otras investigaciones, hemos encontrado que la gran mayoría de noticias recogidas $(75 \%)$ se corresponden con el tipo de informe de caso. Únicamente el 9,5\% de las noticias analizadas son de carácter científico-divulgativo. Hemos detectado diferencias en la manera en que se aborda el tema entre estos dos tipos de publicaciones. En las noticias de informe de caso, hemos observado mayor incumplimiento de las directrices de la OMS: dan cuenta del método utilizado por la persona con conducta suicida; cuando mencionan factores explicativos suelen referir causas simplistas, y en su mayoría no tienen en cuenta los antecedentes en salud mental.

Es en las noticias científico divulgativas en las que se proporciona información sobre ayudas y recursos, se tiene en cuenta el sufrimiento e impacto psicológico sobre la familia, se menciona la relevancia de los antecedentes en salud mental, se explicitan los factores de riesgo más importantes y se ofrecen varios factores expli- 
cativos sobre la conducta suicida. Cuando se publican este tipo de noticias suele buscarse el apoyo y la opinión de expertos, generalmente especialistas en el área de salud mental. Son este tipo de informaciones, por consiguiente, las que tienen en cuenta en mayor medida las recomendaciones realizadas por la OMS, y las que posiblemente pudiesen ayudar a hacer visible el problema del suicidio de manera responsable, contribuyendo a fomentar la prevención del mismo.

En estudios realizados en Japón, Australia y Hungría se hizo evidente un descenso en la tasa de suicidios tras la instauración de guías basadas en las recomendaciones de la OMS y dirigidas, especialmente, a los profesionales de los medios de comunicación $(8,11)$.

Tal como se desprende de los estudios acerca del efecto Papageno, informar acerca del suicidio de la manera adecuada puede tener un efecto preventivo.

El sensacionalismo está presente en el $40 \%$ de los casos según los resultados obtenidos. Aunque por las características del estudio no se puede corroborar que sea más patente cuando se alude a una celebridad, durante la recogida de datos si se pudo observar. En algún caso, incluso el personaje público se vio obligado a desmentir su suicidio (calificado de inexplicable) tras haberse informado en la web de la supuesta hora exacta del mismo, así como del método utilizado.

Posiblemente la repercusión mediática que poseen los personajes famosos influya en que se ofrezcan mayores detalles sensacionalistas a las noticias de las que son protagonistas.

Hemos de mencionar que los estudios llevados a cabo sobre el efecto Werther concluyen que los factores que más influyen sobre el mismo son publicar noticias sobre celebridades en las que se divulgue el método, se ofrezcan explicaciones simplistas y no se tengan en cuenta los antecedentes de la persona en salud mental $(2,5$, $7-8,11)$. No obstante, en la mayor parte de las noticias que hemos analizado sobre celebridades, se explicita el método elegido; cuando se hace mención a las causas suelen ser explicaciones simplistas y no se informa sobre antecedentes psiquiátricos. Apenas se han recogido noticias en las que se incluyan fotografías. Hemos encontrado que en estos casos se trataba de celebridades y que la foto adjuntada era de la persona antes del suceso.

En un solo caso se publicó un vídeo bastante impactante que mostraba el momento de la inmolación de un grupo de monjes tibetanos.

\section{Conclusiones}

El estudio pone de manifiesto que la gran mayoría de las noticias publicadas en la prensa española acerca de suicidio no cumplen con las recomendaciones hechas 
ORIGINALES Y REVISIONES

por la OMS (2000) en el marco del programa SUPRE (SUICIDE PREVENTION) lanzado en el año 1999, siendo las de carácter científico-divulgativo las que más las tienen en cuenta.

No se presenta un cobertura sensacionalista en la mayoría de las noticias aunque sí se realiza cuando se trata de una celebridad.

Las noticias presentan el suicidio mayoritariamente como algo inexplicable o se le atribuyen causas simplistas.

En la mayoría de las noticias nombran o detallan el método utilizado.

La mayor parte de las publicaciones no tienen en cuenta los antecedentes de enfermedad mental de la víctima.

En la gran mayoría de las noticias no se suministra información acerca de ayuda disponible.

Apenas se informa sobre la red de apoyo de la persona.

Sólo en algunos casos, cuando se atribuye la conducta suicida a la crisis, informan del comportamiento suicida como una respuesta entendible a los cambios o a la degradación social o cultural

No se resaltan alternativas al suicidio.

No se informa de los indicadores de riesgo de la conducta suicida.

En España, como en la mayoría de los países de la UE, no existen unas guías de prevención del suicidio dirigidas específicamente a profesionales de los medios de comunicación.

Múltiples estudios realizados en países como Austria, Canadá, Hong Kong o Reino Unido han reportado el beneficio, tanto para profesionales como para la población general, que supone la elaboración de éste tipo de documentos específicos para cada país.

\section{BIBLIOGRAFÍA}

(1) Grupo de trabajo de la Guía de Práctica Clínica de Prevención y Tratamiento de la Conducta Suicida. Guía de Práctica Clínica de Prevención y Tratamiento de la Conducta Suicida. Plan de Calidad para el Sistema Nacional de Salud del Ministerio de Sanidad, Política Social e Igualdad. Agencia de Evaluación de Tecnologías Sanitarias de Galicia (avalia-t); 2012. Guías de Práctica Clínica en el SNS: Avalia-t 2010/02.

(2) Stack, S. The effect of the media on suicide: evidence from Japan, 1955-1985. Suicide and Life-Threatening Behavior. 1996; 26(2): 132-42

(3) Pirkis J, Blood R. Suicide and the media. Part II: Portrayal in fictional media. Crisis (Toronto). 2001; 22(4): 155-162

(4) Goethe JW, Los sufrimientos del joven Werther. Editorial DeBolsillo. 2006

(5) Pirkis J, Blood R. Crisis. Crisis (Toronto). 2001; 22(4): 146-154 
(6) Pirkis J, Blood R. Suicide and the media. Part III: Theoretical issues. Crisis (Toronto). 2001; 22(4): 163-169

(7) Yang AC, Tsai SJ, Yang CH, Shia BC, Fuh JL, Wang SJ et al. Suicide and media reporting: a longitudinal and spatial analysis. Social Psychiatry and psychiatric epidemiology. 2013; 48(3): 427-35

(8) Niederkrotenthaler T, Fu K, Yip P, Fong D, Stack S, Cheng Q et al. Changes in suicide rates following media reports on celebrity suicide: a meta-analysis. Journal of Epidemiology and Community Health. 2012; 66: 1037-1042

(9) Frei A, Schenker T. The Werther effect and assisted suicide. Suicide and Life-Threatening Behavior. 2003; 33(2): 192-200

(10) Organización Mundial de la Salud. Prevención del suicidio: un instrumento para profesionales de los medios de comunicación. Trastornos mentales y cerebrales. Departamento de salud mental y toxicomanías. Ginebra: OMS. 2000

(11) Pirkis J, Blood R. Media guidelines on the reporting of suicide. Crisis (Toronto). 2006; 27(2): $82-7$ 